\title{
Polymorphism in p-Aminobenzoic Acid
}

\author{
Aurora J. Cruz-Cabeza, ${ }^{1,2^{*}}$ Roger J. Davey, ${ }^{1 *}$ Iain D.H. Oswald, ${ }^{3}$ Martin R. Ward, ${ }^{3}$ \\ and Isaac J. Sugden ${ }^{4}$ \\ ${ }^{1}$ School of Chemical Engineering and Analytical Science, University of Manchester, M13 9PL \\ Manchester, United Kingdom. \\ ${ }^{2}$ Astra Zeneca, Silk Road Business Park, Charter Way, Macclesfield, SK10 2NA, United Kingdom. \\ ${ }^{3}$ Strathclyde Institute of Pharmacy \& Biomedical Sciences (SIPBS), University of Strathclyde, 161 \\ Cathedral Street, G4 0RE, Glasgow, United Kingdom. \\ ${ }^{4}$ Molecular Systems Engineering Group, Centre for Process Systems Engineering, Department of \\ Chemical Engineering, Imperial College London, London SW7 2AZ, United Kingdom.
}

Email: aurora.cruzcabeza@manchester.ac.uk; roger.davey@manchester.ac.uk;

\begin{abstract}
We review the polymorphism of p-aminobenzoic acid ( $p \mathrm{ABA}$ ), a model drug compound whose crystallisation and polymorphic behaviour has been extensively studied in recent years. Beyond the well-known and characterised $\alpha$ and $\beta$ forms, $p$ ABA also crystallises as a $\gamma$ polymorph, which is structurally similar to the $\alpha$ form. In addition we also compare the newly reported $\delta$ form, obtained by high pressure crystallisation and through compression of the $\alpha$-form. A structural analysis and comparison of all of the forms is presented, the conditions by which each of them is obtained summarised. Crystal structure prediction calculations have also been carried out in order to probe the solid form energy landscape of this compound. The overall picture of the polymorphism of $p \mathrm{ABA}$, reveals, surprisingly, the rarity of the $\beta$ form.
\end{abstract}

\section{Introduction}

In the field of organic solid-state chemistry the issue of polymorphism continues to be of enormous relevance from both scientific and commercial perspectives. ${ }^{1,2}$ Of particular fundamental importance are issues surrounding the relative nucleation ${ }^{3}$ and growth rates of crystal polymorphs and the use of crystal structure prediction ${ }^{4,5}$ to aid the discovery of new forms in support of commercial applications. In the latter context it is clearly of prime importance to make the link between prediction and experiment for systems which are already proven to be polymorphic.

Here we draw attention to this issue with relation to $p$-aminobenzoic acid ( $p \mathrm{ABA}$ ). Significant work covering crystal morphology, ${ }^{6,7}$ phase behaviour, ${ }^{8}$ nucleation ${ }^{9},{ }^{10}$ and crystal growth kinetics ${ }^{11}$ has been reported over the last decade with reference to two polymorphic forms, $\alpha$ and $\beta$. These forms are related enantiotropically having a transition temperature of $\sim 14{ }^{\circ} \mathrm{C}$ with $\beta$ the stable form below this temperature and $\alpha$ stable above it. ${ }^{12}$ It is known that while solvent crystallisation of the $\beta$ form is actually 
rather difficult, unless water is used, the $\alpha$ polymorph is readily obtained from a range of solvents both above and below the transition temperature. ${ }^{8,13}$ In this context, we set out to review important information available on the polymorphism of $p \mathrm{ABA}$. Finally, we present crystal structure prediction (CSP) calculations for the system and analyse and discuss its crystal energy landscape.

\section{The crystal structures of $p \mathrm{ABA}$ polymorphs.}

The CSD contains 13 entries for pABA (AMBNAC - AMBNAC12). Of these, two structures contain no atomic coordinates (AMBNAC02 and AMBNAC03) ${ }^{14}$ and another three are repeats of existing refcodes with no atomic coordinates (ie. AMBNAC05 repeats AMBNAC04; ${ }^{8,15}$ AMBNAC10 and AMBNAC11 repeat AMBNAC07 and AMBNAC08 ${ }^{6}$ respectively). The remaining refcodes report only 3 unique crystal structures of pABA: the $\alpha, \beta$ and $\gamma$ polymorphs.

Interestingly, given the subsequent apparent difficulties in preparing it, the first reported crystal structure dates from 1966 and corresponds to $\beta$-pABA (AMBNAC). ${ }^{16}$ Following this Lai and Marsh (10) reported the structure of $\alpha-p$ ABA in $1967^{17}$ and noted the prevalence of twinning and disorder within these crystals originating in the randomness of secondary $\mathrm{H}$-bonds $(\mathrm{NH} \ldots \mathrm{O}=\mathrm{C})$ between pABA dimers. Killean et $a l^{14}$ returned to this in AMBNAC03 reporting limited crystallographic data on a form I, which is almost certainly the $\alpha$ form, and two new forms (AMBNAC03) II and III both grown from dioxane. As pointed out later by Kuhnert-Brandstaetter and Grim ${ }^{18}$ these latter forms are most likely solvates. Between 2005 and 2017 there were three new determinations of the $\beta$ (AMBNAC05, ${ }^{15}$ AMBNAC08, ${ }^{6}$ AMBNAC12 ${ }^{19}$ ) and $\alpha$ structures (AMBNAC06, ${ }^{20}$ AMBNAC07, ${ }^{6}$ AMBNAC13 ${ }^{21}$ ). Of the latter it would appear that AMBNAC06 has its amine hydrogens incorrectly positioned so as to make the nitrogen trigonal, rather than the correct pyramidal. In 2014 Benali-Cherif et $a l^{22}$ reported a new noncentric polymorph, $\gamma$, having a dimer motif similar to $\alpha$ but differing slightly in its packing. Reported in the preceding companion paper to this review Ward et al provide details of a new polymorph, $\delta$, produced under high pressure crystallisation conditions. ${ }^{23}$ 
Table 1. Summary of crystallographic information for the four polymorphs of pABA.

\begin{tabular}{|c|c|c|c|c|c|c|c|}
\hline & $\begin{array}{l}\text { CSD REFCODEs } \\
\text { (Year, R factor) }\end{array}$ & $\begin{array}{l}\text { REFCODE } \\
\text { (Temperature) }\end{array}$ & \begin{tabular}{|l|} 
Space \\
Group
\end{tabular} & & $\begin{array}{l}\text { Unit } \\
\text { Cell } \\
\text { Lengths }\end{array}$ & \begin{tabular}{|l} 
Unit \\
Cell \\
Angles
\end{tabular} & $\begin{array}{l}\text { Packing } \\
\text { Coefficient }\end{array}$ \\
\hline$\alpha$ & $\begin{array}{l}\text { AMBNAC01 }(1967,7.0 \%) \\
\text { AMBNAC06 }(2007,6.6 \%) \\
\text { AMBNAC07 }(2014,5.2 \%) \\
\text { AMBNAC13 }(2017,5.1 \%)\end{array}$ & $\begin{array}{l}\text { AMBNAC07 } \\
(101 \mathrm{~K})\end{array}$ & $P 2_{1} / n$ & 2 & $\begin{array}{l}\mathrm{a}: 18.562 \\
\mathrm{~b}: 3.732 \\
\mathrm{c}: 18.568\end{array}$ & $\begin{array}{l}\alpha: 90.0 \\
\beta: 93.9 \\
\gamma: 90.0\end{array}$ & 0.720 \\
\hline$\beta$ & $\begin{array}{l}\text { AMBNAC }(1966,17.0 \%) \\
\text { AMBNAC04 }(2005,5.0 \%) \\
\text { AMBNAC08 }(2014,5.7 \%) \\
\text { AMBNAC12 }(2015,3.0 \%)\end{array}$ & $\begin{array}{l}\text { AMBNAC08 } \\
(102 \text { K) }\end{array}$ & $P 2_{1} / n$ & 1 & $\begin{array}{l}\mathrm{a}: 6.243 \\
\mathrm{~b}: 8.457 \\
\mathrm{c}: 12.365\end{array}$ & $\begin{array}{l}\alpha: 90.0 \\
\beta: 100.2 \\
\gamma: 90.0\end{array}$ & 0.719 \\
\hline$\gamma$ & AMBNAC09 $(2014,5.1 \%)$ & $\begin{array}{l}\text { AMBNAC09 } \\
(100 \mathrm{~K})\end{array}$ & $P n a 2_{1}$ & 2 & $\begin{array}{l}\mathrm{a}: 26.995 \\
\mathrm{~b}: 3.732 \\
\mathrm{c}: 12.673\end{array}$ & $\begin{array}{l}\alpha: 90.0 \\
\beta: 90.0 \\
\gamma: 90.0\end{array}$ & 0.727 \\
\hline$\delta$ & $\begin{array}{l}\text { Ward et al Companion paper }{ }^{23} \\
(2018,2.8 \%)^{\S}\end{array}$ & $\begin{array}{l}\text { Ward et al } \\
(100 \mathrm{~K})\end{array}$ & $P n$ & 1 & $\begin{array}{l}\mathrm{a}: 6.455 \\
\mathrm{~b}: 4.674 \\
\mathrm{c}: 10.547\end{array}$ & $\begin{array}{l}\alpha: 90.0 \\
\beta: 100.7 \\
\gamma: 90.0\end{array}$ & 0.746 \\
\hline
\end{tabular}

*As calculated in Mercury.

${ }^{\S}$ CCDC depostion numbers CCDC 1876686-1876690. Structure at $100 \mathrm{~K}=1876689$.

These data are summarised in Table 1. It is worth making a note on nomenclature here $-\alpha$ and $\beta$ have been variously referred to as I and IV and as discussed above II and III are redundant forms. The CSD refers to the noncentric polymorph as Form V. Given the extent to which $\alpha$ and $\beta$ have been used in the literature we prefer to use these Greek symbols throughout hence the non-centric 2014 form is $\gamma$ and the new form is $\delta$.

\section{Computational Methods.}

CSD Searches, structure visualisation and comparison. Crystal structures of pABA were retrieved from the Cambridge Structural Database (vs. 5.39) ${ }^{24}$ using Conquest. Structures were then visualised in Mercury vs. $3.10 .^{25}$ Polymorphic forms were compared using the Mercury Materials module and the COMPACK algorithm ${ }^{26}$ with standard settings using a molecular cluster size of 20 molecules. PXRD similarity between polymorphs was calculated with the algorithm implemented in Mercury by van de Streek and Motherwell. ${ }^{27}$

\section{Crystal Structure Prediction (CSP).}

CSP calculations for pABA were carried out using the code CrystalPredictor (version 2.3). ${ }^{28-30}$ We were able to sample the crystal packing of pABA efficiently by allowing 
for molecular relaxation around the carboxylic acid group. Appropriately describing for molecular flexibility around the carboxylic acid group was very important for the generation of an accurate initial CSP landscape in pABA. The molecular model used for $p \mathrm{ABA}$ as input to CrystalPredictor was optimised in the gas-phase at the M06 level of theory with a $6-31 G(d, p)$ basis set in Gaussian $09^{31}$ using tight optimization criteria. A local approximate model $(\mathrm{LAM})^{30}$ for the conformational energy, molecular geometry and atomic ESP charges was derived to describe the molecular flexibility around the carboxylic acid group ( $\mathrm{Ph}-\mathrm{COOH}$ torsion) at the same level of theory. This LAM was then used for crystal structure generation and optimisation together with the Williams 99 forcefield (W99) for the description of exchangerepulsion and dispersion interactions. ${ }^{32-34}$

For the structure generation stage, two searches were performed with $Z^{\prime}=1$ and $Z^{\prime}=2$. These sampled, respectively, the $20\left(Z^{\prime}=1\right)$ and the $9\left(Z^{\prime}=2\right)$ most common space groups. 200 and 500k structural minimisations were run for the $Z^{\prime}=1$ and 2 searches respectively. After the CrystalPredictor calculations were completed a final clustering of generated structures was carried out with the COMPACK algorithm. ${ }^{26}$

\section{Improved Lattice Energies: Atomic Multipoles.}

In order to refine the calculated lattice energies, the generated structures were minimised with an improved energy model consisting of the same W99 potential together with an improved model for electrostatics consisting of atomic multipoles. The electron density of the pABA molecule in each crystal structure was recalculated at the M06/6-31G** level of theory in a continuum dielectric environment with a dielectric constant of 2.0 (using a SMD continuum model). ${ }^{35}$ A distributed multipole analysis $^{36,37}$ was then performed on the calculated density in order to derive atomic multipoles. Crystals were then minimized and energies calculated with the software DMACRYS (version 2.1). ${ }^{38}$

\section{Improved Lattice Energies: PBE-d.}

As a final step the lattice energies of the ten most stable crystal structures (together with all the experimental polymorphs) were recomputed with periodic density functional theory with van der Waals corrections. For this, the PBE functional ${ }^{39}$ was used together with PAW pseudopotentials ${ }^{40,41}$ and the Grimme's van der Waals corrections $(\mathrm{d} 2)^{42}$ as implemented in the VASP code (version 5.4). ${ }^{43-46}$ An energy cut 
off of $520 \mathrm{eV}$ was used for the planewaves. The Brillouin zone was sampled using the Monkhorst-Pack approximation ${ }^{47}$ at k-point grids separated by approximately $0.05 \AA$. Crystal structures were relaxed with this model allowing the unit cell volume as well as the atomic positions to optimise. After two optimisation cycles, the energy of the structure was recomputed with the same level of theory. Structural relaxations were halted when the calculated force on every atom was less than $0.003 \mathrm{eV} \AA^{-1}$. Lattice energies $\left(E_{\text {latt }}\right)$ for both forms were calculated by subtracting the electronic energy of a single molecule in the gas-phase from the electronic energy of the simulation cell normalised by the number of molecules $(\mathrm{N})$.

\section{Analyses of the four polymorphs}

\subsection{Overall interactions}

The main type of interactions found in the $p \mathrm{ABA}$ polymorphs are summarised in Figure 1. $p \mathrm{ABA}$ molecules can H-bond through $R_{2}^{2}$ (8) cyclic acid dimers (Fig. 1 top left) or through a carboxylic acid-amino head to tail interaction (Fig. 1 bottom left). Molecules are also involved in aromatic interactions via stacking governed by translation (Fig. 1 top right) or inversion symmetry (Fig. 1 bottom right).

The $\alpha$, and $\gamma$ form have the same type of H-bonding and stacking: $R_{2}^{2}(8)$ acid dimers and stacking related by translation (along the crystallographic $b$ axis in both structures). The $\beta$ and $\delta$ form have the same head to tail H-bonding but whilst stacks in $\beta$ are related by inversion in the new $\delta$ form they are related by translation.

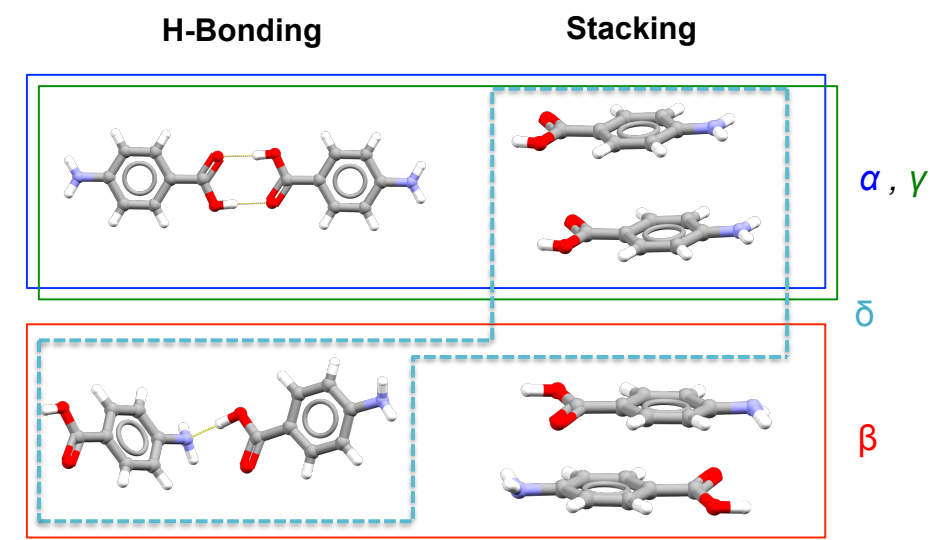

Figure 1. Hydrogen bonding and stacking interactions in the four polymorphs of pABA. 
Further to the main H-bonds and stacking interactions, the $\mathrm{NH}_{2}$ group is also able to get involved in one further H-bond with the carbonyl group. This secondary H-bond is observed in all of the forms (Fig. 2) although the N...O distances vary from 2.94 to $3.3 \AA$.

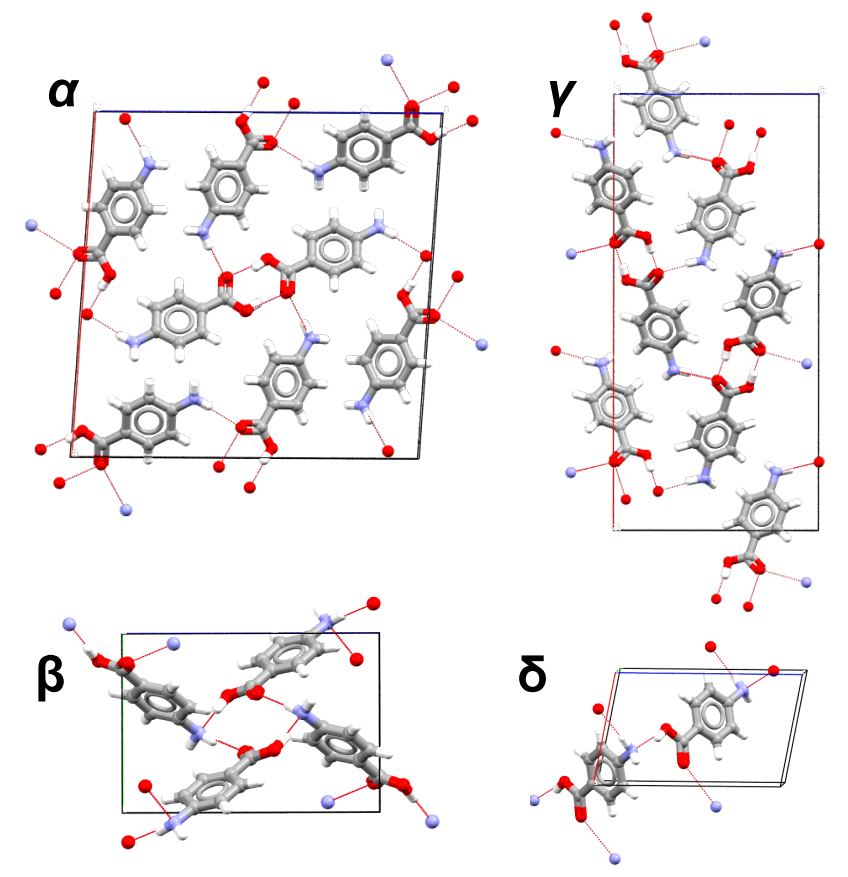

Figure 2. Unit cell view of the four pABA polymorphs.

\section{$4.2 \alpha$ versus $\gamma$}

As already anticipated, the $\alpha$ and the $\gamma$-forms are very similar. $\alpha$ crystallises in a monoclinic space group $\left(P 2_{1} / n\right)$ whilst $\gamma$ crystallises in an orthorhombic space group $\left(P n a 2_{1}\right)$, both with $Z^{\prime}=2$. A COMPACK comparison of the two crystal structures (AMBNAC07 and 09) returns 18 molecules in common out of a molecular shell of 20 molecules.

To understand further the differences between these forms, we can view form $\alpha$ and $\gamma$ as a succession of corrugated layers packed along the [101] direction for form $\alpha$ or the [001] direction for form $\gamma$ (Fig. 3 illustrates form $\alpha$ ). The differences between $\alpha$ and $\gamma$ forms lie in the inner structure of alternate layers. Therefore, the packing of the $\alpha$ and $\gamma$ structures can be understood as a succession of one equivalent and one mismatched layers along the [101] and [001] directions respectively. In an equivalent layer, all atoms are in identical positions (red layers Fig. 3 and left Fig. 4); in a mismatched layer atoms have different arrangements for the $\alpha$ and $\gamma$ structures (right Fig. 4). 


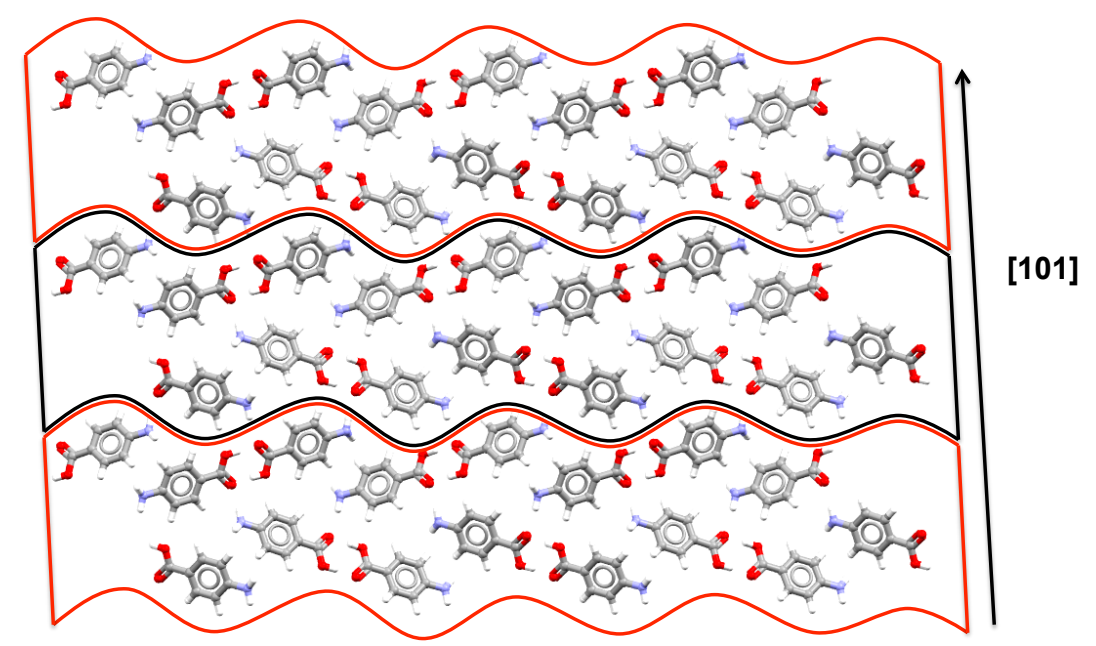

Figure 3. Packing of (101) layers in the $\alpha$ pABA form, view along the b-axis. Black and red wavy lines delineate alternate layers.

Thus the red layers in Figure 3 are equivalent in both forms showing almost identical molecular positions whilst the alternate (black) mismatched layers have molecules orientated in different directions but with centres of mass in almost identical positions. This is seen viewed along [001] in Fig. 4. The (101) surface in the $\alpha$ or the (001) surface in the $\gamma$ form mostly expose the NH2 groups and the side of the carbonyl. So, a different orientation of the $\mathrm{NH}_{2} \ldots \mathrm{O}$ interactions leads these two types of layer orientations characteristic of the $\alpha$ and $\gamma$ forms.
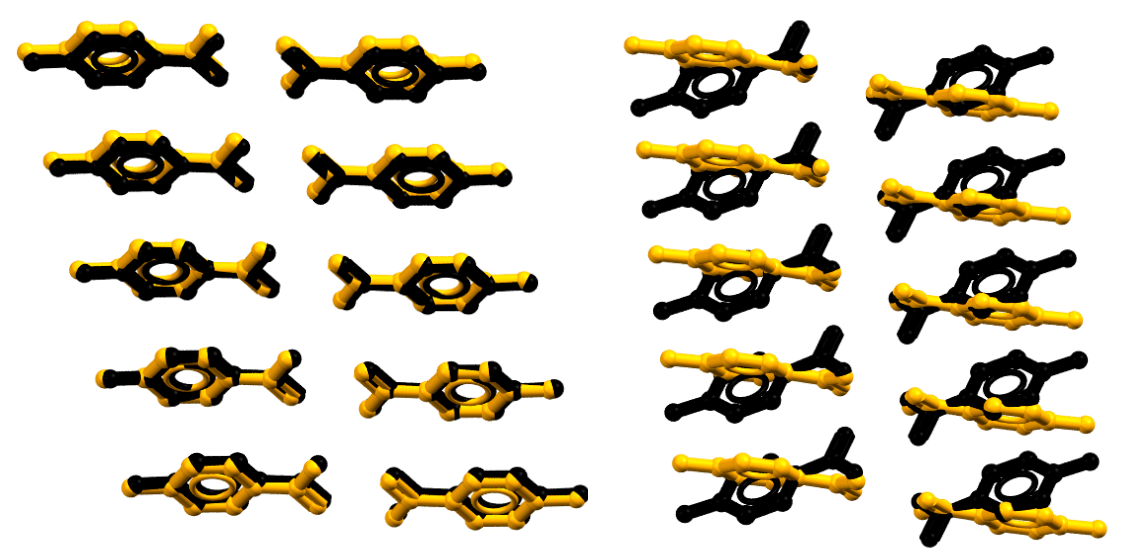

Figure 4. Equivalent layer (left) and mismatched layer (right) in the $\alpha$ (yellow) and $\gamma$ (black) polymorph. View along c.

\section{$4.3 \beta$ versus $\delta$}

The $\beta$ and $\delta$ forms contain identical H-bond head to tail acid-amine dimers. The $\beta$ form crystallises in a $P 2_{1} / n$ structure whilst the $\delta$ form is a $P n$ structure, both with $Z^{\prime}=1$ (Table 1). Thus, whilst $\beta$ is a centric form, $\delta$ is non-centric. Figure 5 shows a 
view of the crystal packing for both forms along the stacking direction. For the noncentric $\delta$ form (Fig. 5, right), the (polar) H-bonded acid-amine chains (O....N- 2.78 $\AA$ ) run roughly along [101] and are stacked by translation along the $b$-axis $(3.5 \AA)$. For the centric $\beta$ form (Fig. 5, left), however, the acid-amide chains are packed in alternate directions allowing them to be joined by the creation of the H-bonded tetramer (Fig. 2) at the expense of the infinite stacking arrangement seen in $\delta$.
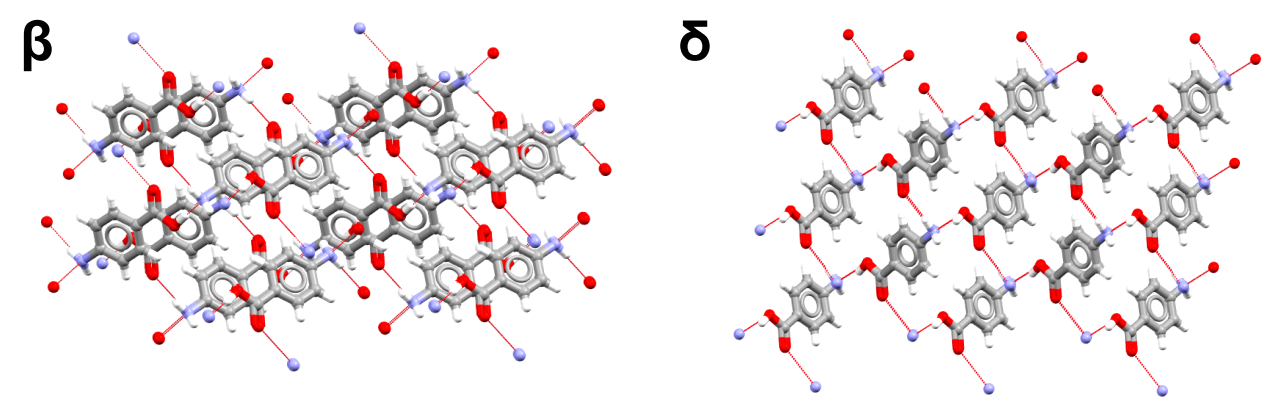

Figure 5. View of the $\beta$ and $\delta$ forms along the stacking direction.

We note of the rarity of the head to tail hydrogen bond motif observed in the $\beta$ and $\delta$ forms of $p \mathrm{ABA}$. A CSD search of the $p$-aminobenzoic acid substructure with any small substituents on the ring carbons 2, 3, 4 and 5, returned nine compounds (CSD refcodes: AMSALA02, AYOSOX, BRABZA01, HOLTAE, PUQFUD, PUQGAK, YOZFIE, YOZFUQ01), all of which crystallise making use of the $R_{2}^{2}(8)$ cyclic acid dimer.

\section{Preparation of the polymorphic forms}

As in many polymorphic systems, crystallisation of some forms is extremely facile whilst others may require very specific crystallisation conditions for their nucleation and growth. From a practical perspective we note that $\alpha-p \mathrm{ABA}$ is the dominant, most accessible polymorph, crystallising under most conditions (solvents and

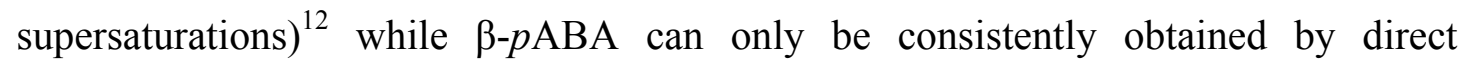
crystallisation from water in a range of supersaturations from $\mathrm{S}=1.1-1.48^{8,13}$ or by phase transformation of an $\alpha-p \mathrm{ABA}$ slurry below $14^{\circ} \mathrm{C}$. Controlled sonication has been reported to aid consistent outcomes in the crystallisation of the $\beta-p \mathrm{ABA},{ }^{48}$ by extending the cooling rates and temperatures over which it is accessible. Whilst $\alpha$ $p$ ABA crystallises in well-known $b$-axis needles (Fig. $6 \mathrm{a}$ and $\mathrm{b}$ ), $\beta$ does so mostly as rhombic blocks though also as plates from ethanol (Fig. 6c). ${ }^{6}$ 
The least reported polymorph is the $\gamma$-form. In their original paper Benali-Cherif et al obtained it as needles from water in the presence of selenous acid. ${ }^{22}$ Given its structural similarity to $\alpha$, it is expected that such needles will also reflect the stacking interaction along the b-axis. More recently Kamali et $a l^{49,50}$ have reported failure to reproduce the crystallisation of the $\gamma$ form from either selenous or phosphinic acids but have found that its preparation using a vapour growth technique is rather facile. Their micrographs show block-like crystals rather than needles, a feature that may be due to the growth conditions employed. As discussed above (section 4.2) and later (Section 8, Fig. 8) the $\alpha$ and $\gamma$ structures are extremely similar and hard to distinguish. Over the last few years the Manchester group have crystallised $\alpha-p$ ABA many hundreds of times from aqueous and organic solutions and we have determined the single crystal structure of the resulting needle crystals on four occasions using both Xray ${ }^{6}$ and neutron diffraction. ${ }^{51}$ Always these had the known $\alpha$ structure in which the two fold rotation axis coincides with the needle b-axis.

Finally, as discussed by Ward et $a l^{23}$ in the preceding companion paper to this, the $\delta$ form is obtained either from direct compression of the $\alpha$-form under high-pressure in pure water or by solution recrystallization also at high pressure. In the former case, $\alpha$ transforms to the new $\delta$ form at a pressure of around $0.85 \mathrm{GPa}$ while in the latter, compression of aqueous $(0.33 \mathrm{GPa})$, aqueous ethanol $(0.8 \mathrm{GPa})$ or ethanolic solutions $(0.49 \mathrm{GPa})$ induced the precipitation of the $\delta$-form. The transition pressure is likely to be close to $0.3 \mathrm{GPa}$ but the pressures quoted are those at which datasets were collected. Single crystals of the $\delta$-form were recoverable to ambient conditions, allowing a full characterisation of the form including XRD and face indexing. The morphology of the $\delta$ - $p \mathrm{ABA}$ crystals obtained at these high pressures is thin plates with dominant (10-1) faces (Fig. 6d). These crystallisation conditions are summarised in Table 2. 


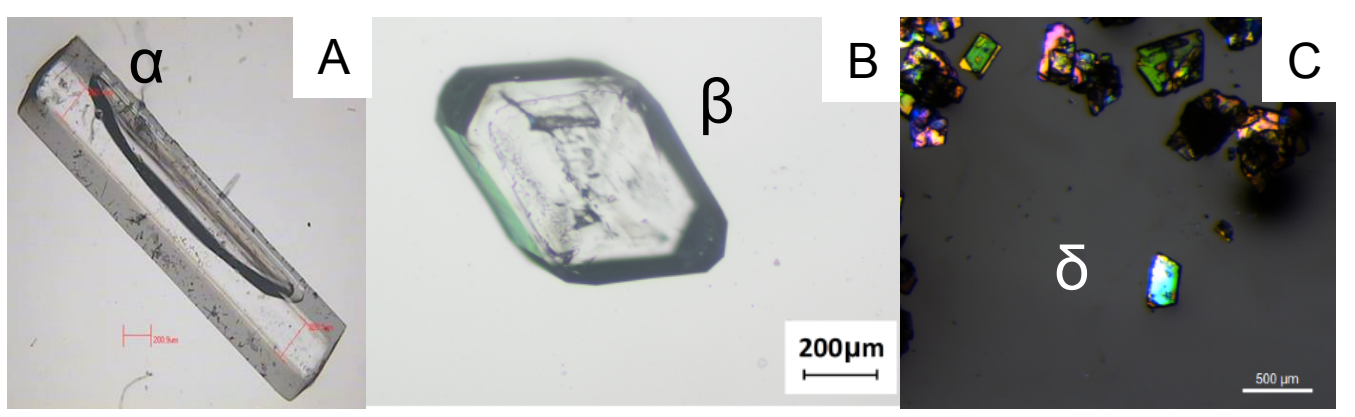

Figure 6. Microscopic images of crystals of $\alpha-p A B A$ grown from methanol $(\mathrm{A})^{6} \beta$ $p$ ABA grown from water $(\mathrm{B})^{13}$ and $\delta$ - $p$ ABA from water at high-pressure $(\mathrm{C}){ }^{23}$

Table 2. Summary of crystallisation conditions which afford the various polymorphs of $p \mathrm{ABA}$.

\begin{tabular}{|l|l|l|l|}
\hline Form & Crystallisation Conditions & Morphology & $\begin{array}{l}\text { Ease of } \\
\text { Crystallisation }\end{array}$ \\
\hline$\alpha$ & $\begin{array}{l}\text { Most crystallisation conditions (solvent and } \\
\text { supersaturation) afford this form. Any temperature. }\end{array}$ & Needles & Dominant \\
\hline$\gamma$ & $\begin{array}{l}\text { From water in the presence of selenous acid/vapour } \\
\text { growth. }\end{array}$ & Needles / Blocks & $\begin{array}{l}\text { Difficult from } \\
\text { solution. }\end{array}$ \\
\hline$\beta$ & $\begin{array}{l}\text { From water at low supersturations only } \\
\text { S }<1.5) . \text { Any temperature. }\end{array}$ & $\begin{array}{l}\text { Blocks / Plates } \\
\text { solution }\end{array}$ \\
\hline$\delta$ & $\begin{array}{l}\text { From water, ethanol and water:ethanol mixtures at high } \\
\text { pressure. }\end{array}$ & Thin plates \\
\hline
\end{tabular}

\section{Nucleation and Growth Rates.}

$p \mathrm{ABA}$ has been the subject of significant numbers of publications focusing on the $\alpha$ and $\beta$ polymorphs. Such work includes measurement of solubility phase diagram in different solvents, the determination of the $\alpha / \beta$ transition temperature, the transformation rates between these two polymorphs and their crystallisation from aqueous and organic solutions. Gracin and Rasmuson ${ }^{8}$ were the first to report a detailed study where the transition temperature between the two forms was determined to be $25^{\circ} \mathrm{C}$ using solubility measurements and the crystallisation conditions for obtaining the $\alpha$ and $\beta$ forms were comprehensively elucidated. It was reported, for the first time that water is the only solvent that can reproducibly yield the $\beta$ form. This point was taken up in some detail by Black et al ${ }^{13}$ who defined precise supersaturation conditions for the isolation of $\beta$ and showed that these were independent of temperature. As far as the $\alpha / \beta$ phase transition temperature is concerned further work by Hao et $a l^{52}$, using solubility combined with slurry experiments, in situ Raman and FTIR and microscopy (FBRM) tools, determined this 
more precisely as $13.8^{\circ} \mathrm{C}$. All such thermodynamic data has been reviewed by Svard et al. ${ }^{12}$

Crystal morphologies of the $\alpha$ and $\beta$ forms were determined through use of goniometry, pXRD and morphological computations by Sullivan and Davey ${ }^{6}$ and this theme was developed in more detail by Rosbottom et $a l^{7}$ who used force field calculations to determine the lattice energies and morphologies of the two forms. They reported the $\alpha$ form to be the more stable by approximately $7.5 \mathrm{kJmol}^{-1}$. The solution mediated interconversion kinetics between the forms were measured by both Hao et $a l^{52}$ and Turner et $a l^{53}$.

As far as kinetic data are concerned Sullivan et $a l^{9}$ reported a detailed study of the nucleation of the $\alpha$ form from a number of organic solvents. The impact of solvent on these rates was confirmed by Turner et al with rate increases from alcohol through acetonitrile to water. ${ }^{54}$ Both studies appeared to highlight the important role of desolvation and hydrogen bonded dimer formation in the crystallisation process. ${ }^{9}$ However later work, in which the solvent dependant nucleation rates of pABA, benzoic, $p$-toluic and $p$-nitrobenzoic acids were compared, revealed that this conclusion was premature and that in fact the rates are controlled by aromatic stacking interactions. More recently, through additional nucleation kinetics measured for $\alpha$-pABA in water and single crystal growth rate measurements on each phase ${ }^{11}$ Black et al have rationalised the labile appearance of $\alpha$ and the role of water in encouraging $\beta$. These data support the view . that the nucleation of these molecules is driven by aromatic stacking rather than H-bond dimer interactions and confirm that that nucleation and growth rates are very closely correlated. ${ }^{55}$ Finally we note very recent work in which through the use of additives chosen to disrupt growth along the stacking direction ( $b$ axis ) of $\alpha$ pABA it has been shown, for the first time, that $\beta$ can be crystallised directly from IPA solutions. ${ }^{56}$

7. Thermal Analysis and Forms Conversions. In their 2004 paper $^{8}$ Gracin and Rasmuson report the transformation and melting behaviour of $\alpha$ and $\beta$ forms. The former melts at between 187 and $189^{\circ} \mathrm{C}$. Crystals of the $\beta$ form transform endothermically to $\alpha$ at temperatures between 70 and $97^{\circ} \mathrm{C}$ (depending on crystal size within the sample). According to the heat of transition rule ${ }^{57}$, this confirms both their enantiotropic relationship and that the true transition temperature $\left(13.8^{\circ} \mathrm{C}\right)$ lies below $70^{\circ} \mathrm{C}$. The heats of fusion and transformation were recorded as $\sim 22.6$ and $2.0 \mathrm{kJmol}^{-1}$. 
In 2008 Yang et $a l^{58}$ confirmed this result and used Raman spectroscopy to measure the rate of the solid state transformation from $\beta$ to $\alpha$ at temperatures between 78 and $100^{\circ} \mathrm{C}$. They observed no intermediate forms and estimated the activation energy to be $136 \mathrm{kJmol}^{-1}$.

Further thermal analysis of $\delta$ and $\gamma$ forms and grinding of the $\delta$ are discussed by Ward et al in the companion paper. ${ }^{23}$ We summarise the form conversions in Figure 7.

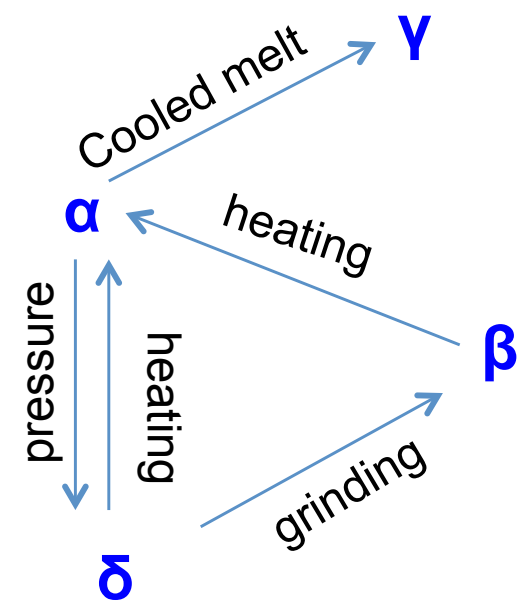

Figure 7. Conversions between forms of pABA.

\section{PXRD fingerprinting of the forms.}

Here we comment on the characterisation of these polymorphs using pXRD. Figure 8 shows the calculated PXRD patterns of the four forms. It is clear that the $\alpha$ and $\gamma$ forms may be easily distinguished from $\beta$ or $\delta$. The $\beta$ and $\delta$ forms show fairly different diffraction patterns. The $\alpha$ and $\gamma$ forms, however, have almost indistinguishable patterns apart from small differences between $2 \theta \sim 24-30^{\circ}$. In fact, the PXRD packing similarity algorithm implemented in Mercury scores these two polymorphs with a PXRD similarity of 0.991 ( 1 being identical). In fact, the structure of the $\gamma$ form is not contained in the best R-factor list for unique crystal structures (given that PXRD similarity is used to distinguish polymorphs in this list). It is plausible, thus, that the $\gamma$ form may have been overlooked given the dominance of $\alpha$. In fact, possible intergrowth of these two forms may explain the twinning and disorder observed by Lai and Marsh ${ }^{17}$ on $\alpha-p$ ABA in 1967 as discussed above in section 2 . 


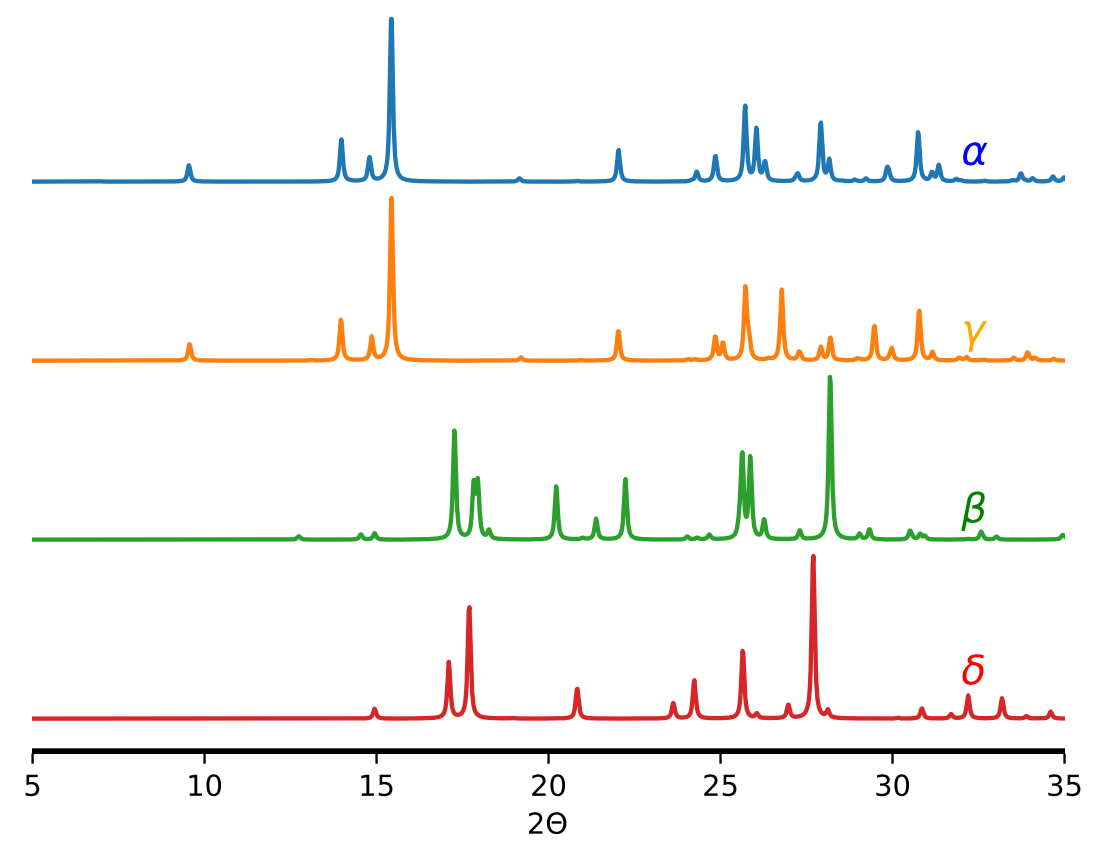

Figure 8. Calculated PXRD patterns for the $p$ ABA polymorphs $(\alpha$ : AMBNAC07, $\gamma$ : AMBNAC09, $\beta$ : AMBNAC08, $\delta$ : this study).

\section{Crystal Structure Prediction \& Computed Stabilities}

As an adjunct to this review of experimental studies crystal structure predictions of pABA were performed to enable further exploration of its solid form landscape. Results are presented in Figure 9 in CSP plots of lattice energy versus packing coefficients for the lowest energy structures within a $10 \mathrm{kJmol}^{-1}$ energy window. The structures are classified according to their H-bonding (left) and stacking (right) type of interactions. Each circle in the plot is a structure generated computationally with a given lattice energy and packing efficiency. A comparison of the experimental forms with the computationally generated structures revealed that, with our CSP model, forms $\alpha, \beta$ and $\gamma$ lie within $1.5 \mathrm{kJmol}^{-1}$ of the global minimum (Fig.9; $\alpha$ : $0.2 \mathrm{kJmol}^{-1}, \beta$ : $0.9 \mathrm{kJmol}^{-1}$ and $\gamma: 1.5 \mathrm{kJmol}^{-1}$ ). The $\delta$ form, obtained under high pressure, was computed to be $5.6 \mathrm{kJmol}^{-1}$ in energy and it corresponds to the densest structure of the entire landscape (Fig. 9).

Of these $\sim 150$ crystal structures generated within $10 \mathrm{~kJ} / \mathrm{mol}$ of the global minimum, all structures except three contain the $R_{2}^{2}(8)$ cyclic acid dimers. Those "unusual" three structures are based on the head to tail COOH...NH2 motif and, remarkably, two of them correspond to experimental forms $\beta$ and $\delta$. 
Analysis of the aromatic stacking motifs also reveals that stacking through translational symmetry is more common than stacking through inversion $(52 \%$ of structures in the landscape stack via translation versus less than $5 \%$ which do so via inversion). We note that the $\beta$ form is in fact a rare occurrence containing both a rare ( $c f$ comment earlier section 4.3) H-bonding motif and stacking interaction based on inversion symmetry.
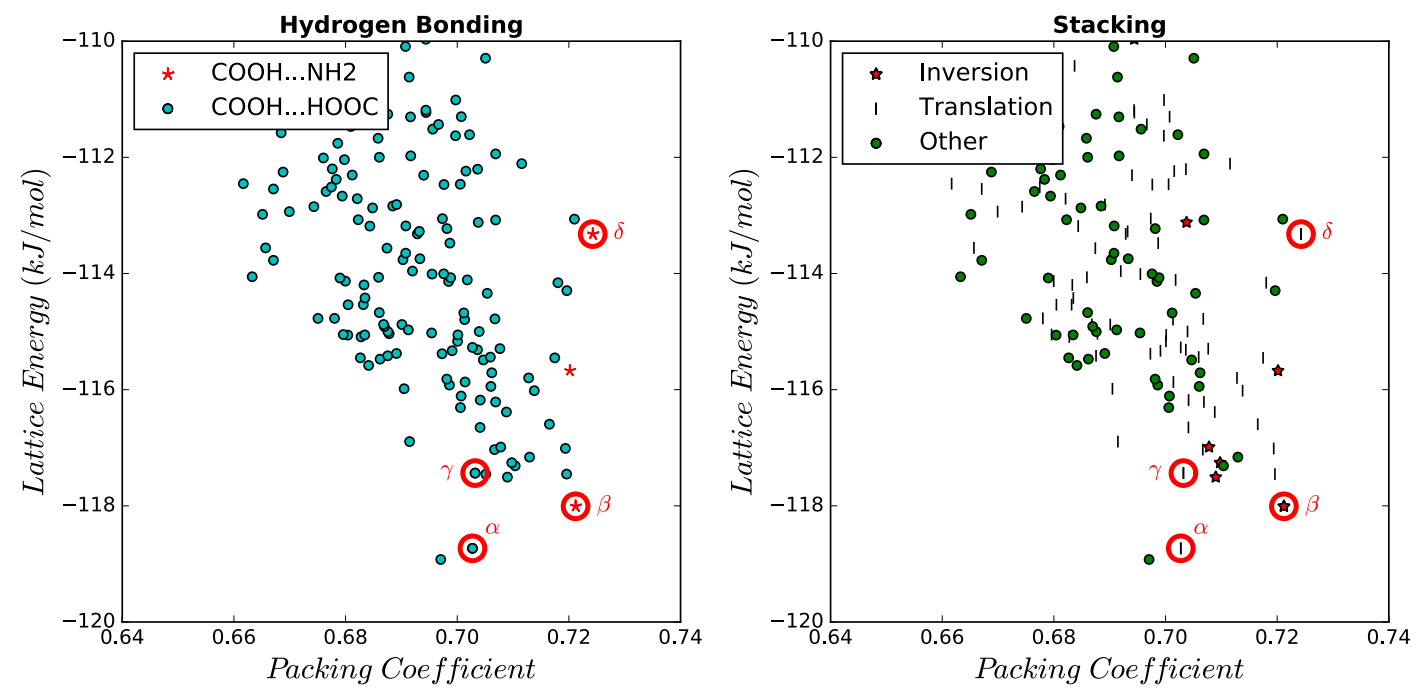

Figure 9. CSP plots with predicted structures classified according to their type of hydrogen bonding (a) and stacking interaction (b).

Further to the CSP study, we recomputed the lattice energy of the lowest energy seven crystal structures (ie. structures within $1.5 \mathrm{kJmol}^{-1}$ of the global minimum, number restricted by computational expense) plus the $\delta$ form. Atomic positions as well as unit cell parameters were allowed to optimise thus enabling optimisation of the $\mathrm{NH}_{2}$ hydrogen atoms positions and adjustments to the pyramidalisation of the nitrogen atom. Modelling nitrogen pyramidalisation is challenging for hybrid models used in CSP and yet this is known to have an important impact in lattice energies. ${ }^{59}$ Thus, for systems containing amines and amides, a final full DFT-d optimisation is a requirement. The results of these calculations are given in Table 3 where it is seen that out of the 7 structures chosen the $\alpha, \gamma, \beta$ and $\delta$ forms became the most stable crystal structures. The relative stabilities of these experimental forms at $0 \mathrm{~K}$ is: $\alpha>\gamma$ $(0.1 \mathrm{~kJ} / \mathrm{mol})>\beta(3.9 \mathrm{~kJ} / \mathrm{mol})>\delta(6.1 \mathrm{~kJ} / \mathrm{mol})$. Unsurprisingly, the $\alpha$ and the $\gamma$ form are computed to be isoenergetic and the $\delta$ form is calculated to be the least stable (without any pressure considerations) but also the structure with the highest density. 
Table 3. Relative stability and packing coefficients of the lowest energy predicted $p$ ABA polymorphs and the delta form as computed with PBE-d.

\begin{tabular}{|c|c|c|c|c|}
\hline Form & $\begin{array}{c}\text { CSP Ranking } \\
\text { (W99 + Multipoles) }\end{array}$ & $\begin{array}{c}\text { Packing } \\
\text { Coefficient }\end{array}$ & $\begin{array}{c}\text { Lattice Energy } \\
\mathbf{( k J / m o l )}\end{array}$ & $\begin{array}{c}\text { Relative Lattice } \\
\text { Energy } \\
(\mathbf{k J} / \mathbf{m o l})\end{array}$ \\
\hline$\alpha$ & 2 & 0.783 & -130.5 & 0.0 \\
\hline$\gamma$ & 7 & 0.785 & -130.4 & 0.1 \\
\hline$\beta$ & 3 & 0.788 & -126.6 & 3.9 \\
\hline$\delta$ & 77 & 0.808 & -124.4 & 6.1 \\
\hline- & 1 & 0.780 & -124.4 & 6.1 \\
\hline- & 5 & 0.791 & -124.1 & 6.4 \\
\hline- & 6 & 0.801 & -123.8 & 6.7 \\
\hline- & 4 & 0.776 & -123.2 & 7.3 \\
\hline
\end{tabular}

Most interestingly, none of the energy models used here or elsewhere ${ }^{7}$ are able to predict $\beta$ as the most stable structure at low temperature. All of the experimental evidence available suggests the $\beta$ form to be the thermodynamically stable form at low temperatures. All of the energy models at $0 \mathrm{~K}$ fail to reproduce this observation, giving $\alpha$ as the more stable of the forms.

\section{Conclusions}

We have reviewed the polymorphism of a popular compound, $p$-aminobenzoic acid, whose crystallisation behaviour has been extensively studied in recent years. Four forms are now known for pABA. Forms $\alpha$ and $\beta$ have been well studied in the past and are easily distinguishable because of their characteristic morphologies (needles for $\alpha$ and chunks for $\beta$ ). They are related enantiotropically with $\beta$ being the low temperature form and a transition temperature at around $14{ }^{\circ} \mathrm{C}$. Whilst the $\alpha$ form appears almost ubiquitously from solution, the $\beta$ can only be crystallised from water and under a very specific set of supersaturations $(\mathrm{S}<1.3)$. Using high pressure together with water and ethanol solutions the new $\delta$ form may be prepared. This new form has some similarities with both the $\alpha$ and the $\beta$ forms; namely $p$ ABA molecules interact via head to tail $\mathrm{COOH} \ldots \mathrm{NH}_{2}$ hydrogen bonds as in $\beta$ and they stack making use of translation symmetry as in $\alpha$. The last form, form $\gamma$, was first reported in 2014 from selenous acid solutions ${ }^{22}$ and it is now known that it can be reproducibly obtained from sublimation. ${ }^{49,50}$ This form has striking similarities with the $\alpha$ form with identical interactions found in the lattices, almost identical PXRD patterns, similar morphologies when grown from solution and being basically isoenergetic 
(differing in energy by only $0.1 \mathrm{~kJ} / \mathrm{mol}$ ). We question whether or not the $\alpha$ and the $\gamma$ form may even be able to intergrow as in the well-known aspirin forms I \& II. ${ }^{60,61}$

Finally, a crystal energy landscape was computed for pABA. The landscape revealed the rarity of the head to tail $(\mathrm{COOH} \ldots \mathrm{NH} 2)$ motif. The experimental polymorphs were computed to be the most stable structures upon DFT-d optimisation of the 7 most stable structures in the landscape. The $\alpha$ form was found to be the most stable with the $\gamma$ form being only $0.1 \mathrm{~kJ} / \mathrm{mol}$ above in energy, then the $\beta(3.9 \mathrm{~kJ} / \mathrm{mol})$ and the $\delta(6.1 \mathrm{~kJ} / \mathrm{mol})$ forms. This stability contradicts the experimental observations of $\beta$ being the most stable form at low temperatures. This highlights the difficulties of measuring and calculating polymorph stabilities accurately even for polymorphs of relatively simple systems.

\section{Acknowledgements}

AJCC thanks the Royal Society for an Industry Fellowship in Astra Zeneca. IDHO \& MRW thank the EPSRC for funding (EP/N015401-1).

\section{References}

1 J. Bernstein, Polymorphism in Molecular Crystals, Oxford University Press, Oxford, New York, 2007.

2 A. J. Cruz-Cabeza, S. M. Reutzel-Edens and J. Bernstein, Chem. Soc. Rev., 2015, 44, 8619-8635.

3 R. J. Davey, S. L. M. Schroeder and J. H. ter Horst, Angew. Chem. Int. Ed., 2013, 52, 2166-2179.

4 A. M. Reilly, R. I. Cooper, C. S. Adjiman, S. Bhattacharya, A. D. Boese, J. G. Brandenburg, P. J. Bygrave, R. Bylsma, J. E. Campbell, R. Car, D. H. Case, R. Chadha, J. C. Cole, K. Cosburn, H. M. Cuppen, F. Curtis, G. M. Day, R. A. DiStasio Jr, A. Dzyabchenko, B. P. van Eijck, D. M. Elking, J. A. van den Ende, J. C. Facelli, M. B. Ferraro, L. Fusti-Molnar, C.-A. Gatsiou, T. S. Gee, R. de Gelder, L. M. Ghiringhelli, H. Goto, S. Grimme, R. Guo, D. W. M. Hofmann, J. Hoja, R. K. Hylton, L. Iuzzolino, W. Jankiewicz, D. T. de Jong, J. Kendrick, N. J. J. de Klerk, H.-Y. Ko, L. N. Kuleshova, X. Li, S. Lohani, F. J. J. Leusen, A. M. Lund, J. Lv, Y. Ma, N. Marom, A. E. Masunov, P. McCabe, D. P. McMahon, H. Meekes, M. P. Metz, A. J. Misquitta, S. Mohamed, B. Monserrat, R. J. Needs, M. A. Neumann, J. Nyman, S. Obata, H. Oberhofer, A. R. Oganov, A. M. Orendt, G. I. Pagola, C. C. Pantelides, C. J. Pickard, R. Podeszwa, L. S. Price, S. L. Price, A. Pulido, M. G. Read, K. Reuter, E. Schneider, C. Schober, G. P. Shields, P. Singh, I. J. Sugden, K. Szalewicz, C. R. Taylor, A. Tkatchenko, M. E. Tuckerman, F. Vacarro, M. Vasileiadis, A. Vazquez-Mayagoitia, L. Vogt, Y. Wang, R. E. Watson, G. A. de Wijs, J. Yang, Q. Zhu and C. R. Groom, Acta Crystallogr. Sect. B Struct. Sci. Cryst. Eng. Mater., 2016, 72, 439-459.

5 A. J. Cruz-Cabeza, Acta Crystallogr. Sect. B Struct. Sci. Cryst. Eng. Mater., 2016, 72, 437-438.

6 R. A. Sullivan and R. J. Davey, CrystEngComm, 2015, 17, 1015-1023.

7 I. Rosbottom, K. J. Roberts and R. Docherty, CrystEngComm, 2015, 17, 5768-5788.

8 S. Gracin and Å. C. Rasmuson, Cryst. Growth Des., 2004, 4, 1013-1023.

9 R. A. Sullivan, R. J. Davey, G. Sadiq, G. Dent, K. R. Back, J. H. ter Horst, D. Toroz and R. B. Hammond, Cryst. Growth Des., 2014, 14, 2689-2696.

10 D. Toroz, I. Rosbottom, T. D. Turner, D. M. C. Corzo, R. B. Hammond, X. Lai and K. J. Roberts, Faraday Discuss., 2015, 179, 79-114.

11 J. F. B. Black, P. T. Cardew, A. J. Cruz-Cabeza, R. J. Davey, S. E. Gilks and R. A. Sullivan, CrystEngComm, 2018, 20, 768-776. 
12 M. Svärd, F. L. Nordström, E.-M. Hoffmann, B. Aziz and Å. C. Rasmuson, CrystEngComm, 2013, 15, 5020-5031.

13 J. F. B. Black, R. J. Davey, R. J. Gowers and A. Yeoh, CrystEngComm, 2015, 17, 5139-5142.

14 R. C. G. Killean, P. Tollin, D. G. Watson and D. W. Young, Acta Crystallogr., 1965, 19, 482-483.

15 S. Gracin and A. Fischer, Acta Crystallogr. Sect. E, 2005, 61, o1242-o1244.

16 M. Alleame, C. Salas-Cimingo and J. Decap, C R Acad Sci Ser C, 1966, 262, 416-417.

17 T. F. Lai and R. E. Marsh, Acta Crystallogr., 1967, 22, 885-893.

18 M. Kuhnert-Brandstaetter and H. Grim, Mikrochim. Acts Wien, 1969, 1208-1209.

19 F. R. Fronczek, CSD Communication 2015.

20 S. Athimoolam and S. Natarajan, Acta Crystallogr. C, 2007, 63, o514-o517.

21 P. P. Nievergelt and B. Spingler, CrystEngComm, 2016, 19, 142-147.

22 R. Benali-Cherif, R. Takouachet, E.-E. Bendeif and N. Benali-Cherif, Acta Crystallogr. Sect. C Struct. Chem., 2014, 70, 323-325.

23 M. Ward, S. Younis, A. J. Cruz-Cabeza, N. Funnell, C. L. Bull and I. Oswald, CrystEngComm. submitted

24 C. R. Groom, I. J. Bruno, M. P. Lightfoot and S. C. Ward, Acta Crystallogr. Sect. B Struct. Sci. Cryst. Eng. Mater., 2016, 72, 171-179.

25 C. F. Macrae, I. J. Bruno, J. A. Chisholm, P. R. Edgington, P. McCabe, E. Pidcock, L. RodriguezMonge, R. Taylor, J. van de Streek and P. A. Wood, J. Appl. Crystallogr., 2008, 41, 466-470.

26 J. A. Chisholm and S. Motherwell, J. Appl. Crystallogr., 2005, 38, 228-231.

27 J. van de Streek and S. Motherwell, Acta Crystallogr. B, 2005, 61, 504-510.

28 P. G. Karamertzanis and C. C. Pantelides, J. Comput. Chem., 2005, 26, 304-324.

29 P. G. Karamertzanis† and C. C. Pantelides, Mol. Phys., 2007, 105, 273-291.

30 M. Habgood, I. J. Sugden, A. V. Kazantsev, C. S. Adjiman and C. C. Pantelides, J. Chem. Theory Comput., 2015, 11, 1957-1969.

31 Gaussian 09, Revision D, M. J. Frisch, G. W. Trucks, H. B. Schlegel, G. E. Scuseria, M. A. Robb, J. R. Cheeseman, G. Scalmani, V. Barone, G. A. Petersson, H. Nakatsuji, X. Li, M. Caricato, A. Marenich, J. Bloino, B. G. Janesko, R. Gomperts, B. Mennucci, H. P. Hratchian, J. V. Ortiz, A. F. Izmaylov, J. L. Sonnenberg, D. Williams-Young, F. Ding, F. Lipparini, F. Egidi, J. Goings, B. Peng, A. Petrone, T. Henderson, D. Ranasinghe, V. G. Zakrzewski, J. Gao, N. Rega, G. Zheng, W. Liang, M. Hada, M. Ehara, K. Toyota, R. Fukuda, J. Hasegawa, M. Ishida, T. Nakajima, Y. Honda, O. Kitao, H. Nakai, T. Vreven, K. Throssell, J. A. Montgomery, Jr., J. E. Peralta, F. Ogliaro, M. Bearpark, J. J. Heyd, E. Brothers, K. N. Kudin, V. N. Staroverov, T. Keith, R. Kobayashi, J. Normand, K. Raghavachari, A. Rendell, J. C. Burant, S. S. Iyengar, J. Tomasi, M. Cossi, J. M. Millam, M. Klene, C. Adamo, R. Cammi, J. W. Ochterski, R. L. Martin, K. Morokuma, O. Farkas, J. B. Foresman, and D. J. Fox, Gaussian, Inc., Wallingford CT, 2016.

32 D. E. Williams, J. Mol. Struct., 1999, 485, 321-347.

33 D. E. Williams, J. Comput. Chem., 2001, 22, 1154-1166.

34 D. E. Williams, J. Comput. Chem., 2001, 22, 1-20.

35 A. V. Marenich, C. J. Cramer and D. G. Truhlar, J. Phys. Chem. B, 2009, 113, 6378-6396.

36 A. J. Stone, Chem. Phys. Lett., 1981, 83, 233-239.

37 A. J. Stone and M. Alderton, Mol. Phys., 1985, 56, 1047-1064.

38 S. L. Price, M. Leslie, G. W. A. Welch, M. Habgood, L. S. Price, P. G. Karamertzanis and G. M. Day, Phys. Chem. Chem. Phys., 2010, 12, 8478-8490.

39 J. P. Perdew, K. Burke and M. Ernzerhof, Phys. Rev. Lett., 1996, 77, 3865-3868.

40 G. Kresse and D. Joubert, Phys. Rev. B, 1999, 59, 1758-1775.

41 P. E. Blöchl, Phys. Rev. B, 1994, 50, 17953-17979.

42 S. Grimme, J. Comput. Chem., 2006, 27, 1787-1799.

43 G. Kresse and J. Furthmüller, Comput. Mater. Sci., 1996, 6, 15-50.

44 G. Kresse and J. Furthmüller, Phys. Rev. B, 1996, 54, 11169-11186.

45 G. Kresse and J. Hafner, Phys. Rev. B, 1993, 47, 558-561.

46 G. Kresse and J. Hafner, Phys. Rev. B, 1994, 49, 14251-14269.

47 H. J. Monkhorst and J. D. Pack, Phys. Rev. B, 1976, 13, 5188-5192.

48 S. Gracin, M. Uusi-Penttilä and Å. C. Rasmuson, Cryst. Growth Des., 2005, 5, 1787-1794.

49 N. Kamali, A. Erxleben and P. McArdle, Cryst. Growth Des., 2016, 16, 2492-2495.

50 N. Kamali, C. O'Malley, M. F. Mahon, A. Erxleben and P. McArdle, Cryst. Growth Des., 2018, 18, 3510-3516.

51 J. Stevens, Private Communication 2018. 
52 H. Hao, M. Barrett, Y. Hu, W. Su, S. Ferguson, B. Wood and B. Glennon, Org. Process Res. Dev., $2012,16,35-41$.

53 T. D. Turner, S. Caddick, R. B. Hammond, K. J. Roberts and X. Lai, Cryst. Growth Des., 2018, 18, 1117-1125.

54 T. D. Turner, D. M. C. Corzo, D. Toroz, A. Curtis, M. M. D. Santos, R. B. Hammond, X. Lai and K. J. Roberts, Phys. Chem. Chem. Phys., 2016, 18, 27507-27520.

55 A. J. Cruz-Cabeza, R. J. Davey, S. S. Sachithananthan, R. Smith, S. K. Tang, T. Vetter and Y. Xiao, Chem. Commun., 2017, 53, 7905-7908.

56 J. F. B. Black, A. J. Cruz-Cabeza, R. J. Davey, R. D. Willacy and A. Yeoh, Cryst. Growth Des., , 2018 18, 7518-7525

57. A. Burger, R. Ramberger, Mikrochim. Acta, 1979, 72, 259-272.

58 X. Yang, X. Wang and C. B. Ching, J. Raman Spectrosc., 2009, 40, 870-875.

59 A. J. Cruz Cabeza, G. M. Day, W. D. S. Motherwell and W. Jones, Cryst. Growth Des., 2006, 6, 1858-1866.

60 A. D. Bond, R. Boese and G. R. Desiraju, Angew. Chem. Int. Ed., 2007, 46, 615-617.

61 A. D. Bond, R. Boese and G. R. Desiraju, Angew. Chem. Int. Ed., 2007, 46, 618-622. 